\title{
Magnetic Properties of Co(II) Complexes with Polyhedral Carborane Ligands.
}

Ofelia B. Oña, ${ }^{\dagger}$ Diego R. Alcoba, ${ }^{\dagger} \uparrow$ Gustavo E. Massaccesi, ${ }^{\S}$ Alicia Torre," Luis Lain," Juan I. Melo, ${ }^{+,}$Josep M. Oliva-Enrich, ${ }^{\perp}$ and Juan E. Peralta*,\#

$\dagger$ Instituto de Investigaciones Fisicoquímicas Teóricas y Aplicadas, Universidad Nacional de la Plata, CCT La Plata, Consejo Nacional de Investigaciones Científicas y Técnicas, Diag. 113 y 64 (s/n), Sucursal 4, CC 16, 1900 La Plata, Argentina

$\ddagger$ Departamento de Física, Facultad de Ciencias Exactas y Naturales, Universidad de

Buenos Aires, Ciudad Universitaria, 1428, Buenos Aires, Argentina

IInstituto de Física de Buenos Aires, Consejo Nacional de Investigaciones Científicas y

Técnicas, Ciudad Universitaria, 1428, Buenos Aires, Argentina

$\S$ Departamento de Ciencias Exactas, Ciclo Básico Común, Universidad de Buenos Aires, Ciudad Universitaria, 1428, Buenos Aires, Argentina

||Departamento de Química Física. Facultad de Ciencia y Tecnología. Universidad del País Vasco. Apdo. 644 E-48080 Bilbao, Spain

$\perp$ Instituto de Química Física "Rocasolano", Consejo Superior de Investigaciones Cientificas, 28006 Madrid, Spain

\#Department of Physics, Central Michigan University, Mount Pleasant, Michigan 48859, USA

E-mail: juan.peralta@cmich.edu

\begin{abstract}
In this work we present a computational analysis of a new family of magnetic $\mathrm{Co}(\mathrm{II})$
\end{abstract}


single-ion complexes with large magnetic anisotropy based on icosahedral and octahedral carborane ligands. In particular, we extend our previous computational work [Alcoba et al. Inorg. Chem. 57, 7763 (2018)] on mononuclear Co(II) complexes with 1,2-(HS) $)_{2}-1,2-\mathrm{C}_{2} \mathrm{~B}_{10} \mathrm{H}_{10}$ and $9,12-(\mathrm{HS})_{2}-1,2-\mathrm{C}_{2} \mathrm{~B}_{10} \mathrm{H}_{10}$ icosahedral $o$-carborane ligands to a larger set of complexes where the $\mathrm{Co}(\mathrm{II})$ ion is doubly chelated by those ligands and by other two positional isomers belonging to the 1,2-dicarba-closo-dodecaborane family. We also describe $\mathrm{Co}(\mathrm{II})$ complexes with octahedral ligands derived from 1,2dicarba-closo-hexaborane and study the effects of replacing a thiol group by a hydroxy one in both polyhedral geometries, as well as the influence of the position of the carbon atoms. Analyzing the results for a total of 20 complexes, our results show that carborane-based $\mathrm{Co}(\mathrm{II})$ single-ion compounds present a distorted tetrahedral geometry, high spin ground states, and high values for the magnetic anisotropy parameters. We point out which of these would be suitable candidates to be synthesized and used as molecular magnets.

Keywords: Carboranes $\cdot \mathrm{Co}(\mathrm{II})$ complexes $\cdot$ Single-molecule magnets $\cdot$ Magnetic anisotropy - Spin relaxation

\section{Introduction}

The magnetic properties of molecular complexes, crystals, and aggregates have received a lot of attention and motivated significant research in this area in the past decades ${ }^{1-4}$ with potential applications in quantum computing, spintronics, energy storage, and other applications. ${ }^{5-8}$ The characteristics of the magnetic properties depend ultimately on the internal

electronic structure of the systems under consideration. Transition metal (TM) complexes play a significant role among complexes with potentially useful magnetic properties for practical applications. ${ }^{9,10}$ These molecules consist of TM ions with high-spin ground states stabilized by inorganic or organic ligands, and their magnetic properties depend largely on the electronic structure of the metal-ligand assembly. Some TM complexes present magnetic 
hysteresis at low temperatures and, consequently, they can be regarded as single molecule magnets (SMM). ${ }^{11}$ One of the characteristics of SMMs is that they show slow magnetic relaxation, that is, the persistence of a net magnetic moment for a long time below a blocking temperature. ${ }^{12}$ In most cases, this originates in the presence of a strong spin-orbit coupling, which can be characterized in model spin-Hamiltonians by two magnetic anisotropy parameters: the axial $(D)$ and the rhombic $(E) .{ }^{13,14}$ The action of an external magnetic field (Zeeman effect) splits the otherwise degenerate spin-orbit substates, and thus imbalances their populations. Removing the external magnetic field leads to a relaxation of the spin system to restore equilibrium. If the energy barrier between the two degenerate states is large, the relaxation is slow and magnetic hysteresis is observed. In order to harness the potential properties of molecular complexes as SMMs, it is therefore reasonable to pursue the experimental synthesis of complexes with combinations of TM ions and ligands that lead to large magnetic anisotropy barriers and hence high blocking temperatures. In this aspect, electronic structure calculations provide valuable information by pointing to potential candidates that can be later synthesized in the laboratory.

Carborane-derived complexes have proven to possess peculiar properties related to photochemistry, catalysis and molecular magnetism. ${ }^{15-17}$ In particular, closo-dodecaborane and closo-hexaborane complexes are icosahedral and octahedral structures, respectively, composed of boron hydrides and carbon atoms forming triangulated polyhedra. These complexes consist typically of one or several units connected by bridge molecules. ${ }^{18-21}$ Carborane complexes have been employed in a large number of applications, but to the best of our knowledge, there have been few attempts to introduce carboranes as SMMs. One of these recent works reports a mononuclear $\mathrm{Co}(\mathrm{II})$ complex with o-carborane dithiolate ligands that displays SMM properties and has been synthesized from $\mathrm{CoCl}_{2} \cdot 6 \mathrm{H}_{2} \mathrm{O}$ and 1,2dithiol-o-carborane. ${ }^{22}$ Experimental studies on the structure of this complex show that two o-carborane-1,2-dithiolate ligands chelate the $\mathrm{Co}(\mathrm{II})$ central ion, arranged as a distorted tetrahedral coordination geometry. Ref. 22 also reports a large magnetic anisotropy, 
with slow zero field magnetic relaxation and magnetic hysteresis at $1.8 \mathrm{~K}$. Very recently we have performed a computational characterization of this complex ${ }^{23}$ that was in good agreement with experimental observations and served as a validation of the computational methodology employed. Two other isomers, derived from the ligands 1,2-(HS) $)_{2}-1,2-\mathrm{C}_{2} \mathrm{~B}_{10} \mathrm{H}_{10}$ and 9,12-(HS) $)_{2}-1,2-\mathrm{C}_{2} \mathrm{~B}_{10} \mathrm{H}_{10}$ were also considered in that work. Our calculations revealed that these complexes show different axial and rhombic magnetic anisotropy parameters as well as different components of the Zeeman anisotropy tensor $\mathrm{g} .{ }^{8}$ The axial anisotropy obtained for one of these three isomers was twice as large as that observed experimentally in Ref. 22, suggesting that polyhedral carborane ligands can be used to control the magnetic anisotropy in these complexes. The purpose of this work is to characterize and gain insight into the magnetic properties of a larger family of mononuclear $\mathrm{Co}(\mathrm{II})$ complexes with polyhedral carborane ligands from first principles electronic structure calculations. To this end, we consider two sets of structures, where different ligands chelate the $\mathrm{Co}(\mathrm{II})$ center to form a distorted tetrahedral coordination geometry. The first set of structures is composed of 1,2-dithiol-o-dodecaborane ${ }^{24,25}$ and 9,12-dithiol-o-dodecaborane ${ }^{26}$ ligands (which have been recently explored by us in Ref. 23), and from building-block units such as 1,2-dihydroxy-o-dodecaborane ${ }^{27}$ and 9,12-dihydroxy-o-dodecaborane. The other set is constructed from building-block units such as 1,2-dithiol-o-hexaborane, ${ }^{28}$ 4,6-dithiol-ohexaborane, 1,2-dihydroxy-o-hexaborane ${ }^{28}$ and 4,6-dihydroxy-o-hexaborane. All these structures are shown in Figure 1.

\section{Theoretical and Computational Aspects}

In this work we characterize the magnetic properties of the aforementioned $\mathrm{Co}(\mathrm{II})$ complexes by means of an effective spin Hamiltonian $\hat{H}^{\text {eff }}$, which in an appropriate coordinate system can be formulated as ${ }^{8,29,30}$ 


$$
\hat{H}^{e f f}=D\left[\hat{S}_{z}^{2}-\frac{S(S+1)}{3}\right]+E\left(\hat{S}_{x}^{2}-\hat{S}_{y}^{2}\right)+\mu_{B} \mathbf{B} \cdot \mathbf{g} \cdot \hat{\mathbf{S}}
$$

where $D$ and $E$ are the axial and rhombic magnetic anisotropy parameters and $\hat{S}_{x}, \hat{S}_{y}$, and $\hat{S}_{z}$ are the Cartesian components of the effective spin operator $\hat{\mathbf{S}}$. The third term in the r.h.s. of Eq. 1 represents the interaction with an external magnetic field $\mathbf{B}$, where $\mu_{B}$ is the Bohr magneton and $\mathbf{g}$ is the Zeeman anisotropy tensor. The spin states are represented by $\left|S, M_{S}\right\rangle$, where $S$ and $M_{S}$ are the standard main and magnetic spin quantum numbers, respectively.

For a better understanding of the relation between calculated magnetic properties and the structures of these complexes, we analyze deformations of the polyhedral structure with the ligands using a simple yet general method known as the minimum bounding ellipsoid (MBE) scheme, as recently described in Ref. 31. The vertices of high symmetry polyhedra lie on a spherical surface. According to the MBE method, distortions of the polyhedra transform that sphere into an ellipsoid which is quantitatively described by the MBE. This scheme allows one to analyze structural data of a TM ion and its environment using only a few parameters that are sensitive to both distance changes and angle distortions. Within this method, the principal axes of the ellipsoid $\left(R_{1} \geq R_{2} \geq R_{3}\right)$ and their mean value $<R>$ are related to the polyhedron size, while the off-center displacement of the central atom $(d)$ and the standard deviation $\sigma(R)$ account for its distortion. The quantity $\mathrm{S}=R_{3} / R_{2}-R_{2} / R_{1}$ is a measure of the ellipsoid shape: $\mathrm{S}<0$ corresponds to oblate (axially compressed), $\mathrm{S}>0$ to prolate (axially stretched), and $\mathrm{S}=0$ to perfect sphericity.

The geometrical structures of the first set of complexes were built from 1,2-dithiolo-dodecaborane and 9,12-dithiol-o-dodecaborane ligands as well as from 1,2-dihydroxy-ododecaborane and 9,12-dihydroxy-o-dodecaborane ones (Figure 2). The second set was constructed from 1,2-dithiol-o-hexaborane and 4,6-dithiol-o-hexaborane ligands and from 1,2- 
dihydroxy-o-hexaborane and 4,6-dihydroxy-o-hexaborane (Figure 3). All calculations were carried out using the computational framework recently described in Ref. 23. The gas-phase structures of all complexes were fully relaxed using density functional theory (DFT) within the BP86 approximation and the def2-TZVP basis sets with the resolution of the identity (RI) approximation and the corresponding auxiliary basis functions. ${ }^{32-36}$ All anisotropy parameters were calculated using the complete active space self-consistent field (CASSCF) method, with an active space of seven electrons distributed over five $3 d$ orbitals, giving rise to ten $S=3 / 2$ and forty $S=1 / 2$ configurations. $N$-electron valence perturbation theory (NEVPT2) $)^{37-39}$ calculations have been performed to account for dynamic correlation on top of CASSCF-converged wave functions. We used quasi-degenerated perturbation theory (QDPT) in combination with the Breit-Pauli approximation for the spin-coupling operator to calculate zero-field splitting (ZFS) parameters. ${ }^{40}$ The molar magnetic susceptibility and the magnetization were evaluated by differentiation of the QDPT Hamiltonian. All calculations were carried out using the ORCA package. ${ }^{41}$

\section{Results and Discussion}

In the following two subsections we present the results derived from 1,2-dicarba-closo-dodecaborane and 1,2-dicarba-closo-hexaborane as reference structures. In all Tables and Figures the labels $\mathrm{A}, \mathrm{B}$ and $\mathrm{C}$ refer to the relative orientation of the carbon atoms of one ligand, while the subscript 0,2 , and 4 , is the number of sulfur atoms which have been replaced by oxygen atoms in the corresponding structure.

\section{1,2-dicarba-closo-dodecaborane Structures}

The structures of complexes constructed from 1,2-dicarba-closo-dodecaborane are shown in Figure 2 and their main geometrical features are given in the Supporting Information. Bond lengths and bond angles between two atoms of the carborane cage with the Co(II) center, 


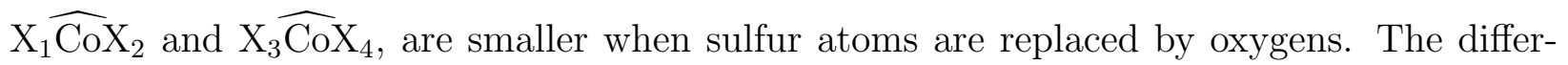
ent combinations of $o$-carborane-1,2-dithiolate, $o$-carborane-9,12-dithiolate, $o$-carborane-1,2hydroxylated and o-carborane-9,12-hydroxylated ligands chelate the $\mathrm{Co}(\mathrm{II})$ center to form a slightly distorted tetrahedron. Consequently, the Co(II) environment differs from the ideal free-ion tetrahedral $\left(\mathrm{T}_{\mathrm{d}}\right)$ symmetry and produces the splitting of the ${ }^{4} \mathrm{~F} \mathrm{Co}(\mathrm{II})$ term, which originates the different $D$ values found for tetrahedral $\mathrm{Co}(\mathrm{II})$ complexes. Calculated $D$ and $E / D$ at the CASSCF/NEVPT2 level of theory are reported in Table 1 . The values found for the $D$ parameter increase for complexes of type A when sulfur is substituted by oxygen, while these values decrease for complexes of type B and C. Rhombicity in the D-tensor and g-tensor for the Ground-state Kramers Doublet (gGKD) is observed in $\mathrm{B}_{2}^{\prime}, \mathrm{C}_{2}$, and $\mathrm{B}_{4}$ with $E / D$ larger than 0.01 , indicating the presence of non-uniaxial anisotropy in the Kramers doublet $(\mathrm{KD})$. On the other hand, for the rest of the complexes, the very small $E / D$ values confirm a high axial anisotropy.

In order to gain additional insight into the electronic structure and magnetic properties of the complexes considered in this work, we have analyzed the influence of both static and dynamic electronic correlation in our calculations. The computed excitation energies are collected in Table 2. Before including the spin-orbit interaction, we have found $S=$ $3 / 2$ ground states in all cases. In these conditions, our results indicate the presence of low-lying spin-orbit-free excited states with very close energies $(\delta)$ above the ground state for compounds $\mathrm{B}_{0}$ and $\mathrm{A}_{2}$, showing an inverse correlation between such energy difference and the calculated $D$ value. The low-lying spin-orbit-free excited states of the remaining complexes are at least $600.0 \mathrm{~cm}^{-1}$ above the ground state. With the inclusion of spin-orbit in the calculations, a set of KDs is obtained, with one low-lying KD of energy $\Delta$ about 87.1-294.4 $\mathrm{cm}^{-1}$ above the ground state for complexes without sulfur substitutions, 81.4$285.3 \mathrm{~cm}^{-1}$ with two sulfur substitutions, and $77.5-176.4 \mathrm{~cm}^{-1}$ with four sulfur substitutions, possibly explaining the spin relaxation processes. The small (large) negative $D$ values in all complexes imply small (large) energy gaps $(\Delta)$. The second KD appears at higher energies, 
635.5-1661.2 $\mathrm{cm}^{-1}$ for complexes without sulfur substitutions, $659.2-1758.0 \mathrm{~cm}^{-1}$ with two sulfur substitutions, and 992.8-1798.5 $\mathrm{cm}^{-1}$ with four sulfur substitutions, and, consequently, they do not contribute to the spin-relaxation mechanism. The $\delta$ energy for complexes $\mathrm{B}_{0}$ and $\mathrm{A}_{2}$ is 68.5 and $167.6 \mathrm{~cm}^{-1}$, respectively, and could lead to an overestimation of the calculated $D$. In order to provide a direct comparison with future experiments, in the Supporting Information section we provide a list of excitation energies for all complexes that allows one to compare data of the temperature dependence of the magnetic susceptibility and the field dependence of the molar magnetization. ${ }^{8,23}$

To correlate the influence of the structural changes between isomers with and without different sulfur substitutions on the $D$ parameter, we performed an ellipsoidal analysis of the $\mathrm{X}_{1} \mathrm{X}_{2} \mathrm{CoX}_{3} \mathrm{X}_{4}$ polyhedra (see Figure 2) using the MBE method based on the Khachiyan procedure. ${ }^{42}$ This method serves to quantify the deviation from the perfect tetrahedral symmetry of these complexes (Table 3). From this analysis, we find a correlation between the standard deviation $\sigma(R)$ and the calculated $D$ parameters whenever the $\sigma(R)$ difference between two isomers (with identical chemical environment for the central Co(II) atom) is greater than approximately $0.03 \AA$. When the difference in the $\sigma(R)$ of two isomers is less than $0.03 \AA$, the highest displacement $d$ of the central atom $\mathrm{Co}(\mathrm{II})$ relative to the ellipsoid center corresponds to the complex with the highest $D$ (in absolute value). These simple empirical rules can be used then to sort isomers according to their $D$ parameter and eventually to be able to identify isomers of high magnetic anisotropy.

Calculations in the basis of the ten $S=3 / 2$ and forty $S=1 / 2$ multiplets at the CASSCF/NEVPT2 level, including spin-orbit contribution, allow us to compute $\chi T(\chi$ is the molar magnetic susceptibility and $T$ the absolute temperature) and the molar magnetization, $M,(M=\chi B)$. Table 4 shows calculated $\chi T$ at 3.0 and $300.0 \mathrm{~K}$ at 1000 Oe, and $M$ at $2.0 \mathrm{~K}$ and $7 \mathrm{~T}$. Additional data are presented in the Supporting Information. $\chi T$ presents a linear behavior at low $T$ and saturates at higher $T$, according to Curie law. $\chi T$ at $300.0 \mathrm{~K}$ is in the value range of $2.875-3.571 \mathrm{~cm}^{3} \mathrm{~mol}^{-1} \mathrm{~K}$ for complexes without sul- 
fur substitutions, 2.850-3.570 $\mathrm{cm}^{3} \mathrm{~mol}^{-1} \mathrm{~K}$ for complexes with two sulfur substitutions, and 2.861-3.304 $\mathrm{cm}^{3} \mathrm{~mol}^{-1} \mathrm{~K}$ for complexes with four sulfur substitutions. These values turn out to be higher than the expected $1.875 \mathrm{~cm}^{3} \mathrm{~mol}^{-1} \mathrm{~K}$ for one isolated $\mathrm{Co}(\mathrm{II})$ ion $(S=3 / 2)$ center with $g=2$, although they lie in the interval $2.1-3.4 \mathrm{~cm}^{3} \mathrm{~mol}^{-1} \mathrm{~K}$, which is typical for a single high spin $d^{7} \mathrm{Co}(\mathrm{II})$ ion configuration with high orbital angular momentum contribution (except in $\mathrm{B}_{0}$ and $\left.\mathrm{A}_{2}\right) . \chi T$ is roughly constant at high temperatures $(300.0-100.0 \mathrm{~K})$, but it reduces to $2.087-3.024 \mathrm{~mol}^{-1} \mathrm{~K}$ without sulfur substitutions, $2.030-3.012 \mathrm{~mol}^{-1} \mathrm{~K}$ with two sulfur substitutions and 2.042-2.626 $\mathrm{mol}^{-1} \mathrm{~K}$ with four sulfur substitutions at $3.0 \mathrm{~K}$, what is due to the splitting undergone by the $\mathrm{Co}(\mathrm{II})$ ion at zero field. In all cases, the decrease of the $\chi T$ product is most likely a consequence of the magnetic anisotropy and/or thermal depopulation of the excited states. The magnetization of these complexes shows a qualitatively similar magnetic behavior. In all cases, the magnetization curves reveal a rapid and steady increase of the magnetization at $2.0 \mathrm{~K}$ without clear saturation at $7 \mathrm{~T}$. Calculated magnetizations range from 2.181 to $2.494 \mu_{B}$ without sulfur substitutions, 2.204 to $2.492 \mu_{B}$ with two sulfur substitutions, and 2.181 to $2.356 \mu_{B}$ with four sulfur substitutions. These values are considerably lower than the expected saturation value for $S=3 / 2$ ion at $7 \mathrm{~T}$ and $2.0 \mathrm{~K}\left(M_{\mathrm{sat}}=3.88 \mu_{B}\right)$, showing the presence of a large zero-field splitting. ${ }^{43}$

\section{1,2-dicarba-closo-hexaborane structures}

Structural data of the fully optimized structures constructed from 1,2-dicarba-closo-hexaborane are shown in the Supporting Information. Bond lengths and bond angles of these complexes are longer than their 1,2-dicarba-closo-dodecaborane counterparts. Similarly to complexes built from 1,2-dicarba-closo-dodecaborane, the different combinations of ligands chelate the $\mathrm{Co}(\mathrm{II})$ center to form a slightly distorted tetrahedron. The $D$ and $E / D$ values calculated at CASSCF/NEVPT2 level are reported in Table 5. Most of the complexes show D- and gGKD-tensors with rhombohedral symmetry, except $\mathrm{A}_{0}$ where $E / D$ is almost zero with high

axial anisotropy. The calculated $D$ parameter increases in absolute value for complexes of 
type $\mathrm{C}$ when sulfur atoms are substituted by oxygen, in contrast to complexes of type A and $\mathrm{B}$, where the absolute value of $D$ decreases. For $\mathrm{C}_{2}$, the $D$ parameter is positive, indicating that the $m_{S}= \pm 1 / 2 \mathrm{KD}$ is below in energy than the reference unperturbed state. Table 6 shows the calculated structural parameters using the MBE method. As in the case of complexes constructed from 1,2-dicarba-closo-dodecaborane, we found an identical empirical relation between the geometrical parameters $\sigma(R)$ and $d$ and the $D$ parameter that can be used to sort isomers according to their magnetic anisotropy.

The electronic structure, as described in the previous subsection, is slightly affected when hexaborane ligands replace dodecaborane ones (see $\Delta$ energies in Table 7 ). In all cases, a $S=3 / 2$ ground state was found before including spin-orbit effects. Only the complex $\mathrm{A}_{0}$ shows a first excited energy $\left(\delta=146.2 \mathrm{~cm}^{-1}\right)$ close to the ground state, which could lead to an overestimation of the calculated $D$. More details can be found in the Supporting Information. The remaining complexes have higher excitation energies of at least $880.0 \mathrm{~cm}^{-1}$ above the ground state, higher than those corresponding to complexes built from 1,2-dicarba-closododecaborane.

Similarly to the complexes with 1,2-dicarba-closo-dodecaborane shown in the previous subsection, $\chi T$ has a linear behavior at low $T$, while it saturates at higher $T$. In these complexes, the $\chi T$ value at $300.0 \mathrm{~K}$ and 1000 Oe is in the range of $2.771-3.560 \mathrm{~cm}^{3} \mathrm{~mol}^{-1} \mathrm{~K}$ for complexes without sulfur substitutions, 2.770-3.182 $\mathrm{cm}^{3} \mathrm{~mol}^{-1} \mathrm{~K}$ for complexes with two sulfur substitutions, and 2.760-3.085 $\mathrm{cm}^{3} \mathrm{~mol}^{-1} \mathrm{~K}$ for complexes with four sulfur substitutions (see Table 8 and Supporting Information). These values are higher than the expected $1.875 \mathrm{~cm}^{3} \mathrm{~mol}^{-1} \mathrm{~K}$ for an isolated $\mathrm{Co}(\mathrm{II})$ ion $(S=3 / 2)$ with $g=2$, but lie (in most cases except for $\mathrm{A}_{0}$ ) in the interval $2.1-3.4 \mathrm{~cm}^{3} \mathrm{~mol}^{-1} \mathrm{~K}$, which is characteristic of a single noninteracting high spin $\mathrm{Co}(\mathrm{II})$ ion in a $d^{7}$ configuration and high orbital angular momentum contribution. $\chi T$ is roughly constant in the high temperature interval $(300.0-100.0 \mathrm{~K})$, then decreases to $1.861-3.016 \mathrm{~mol}^{-1} \mathrm{~K}$ without sulfur substitutions, $1.792-2.495 \mathrm{~mol}^{-1} \mathrm{~K}$ with two sulfur substitutions, and 1.856-2.350 $\mathrm{mol}^{-1} \mathrm{~K}$ with four sulfur substitutions at $3.0 \mathrm{~K}$, which 
is due to the splitting of the $\mathrm{Co}(\mathrm{II})$ ion at zero field. The magnetization at $7 \mathrm{~T}$ and $2.0 \mathrm{~K}$ is 2.203-2.495 $\mu_{B}$ without sulfur substitutions, 2.206-2.435 $\mu_{B}$ with two sulfur substitutions, and 2.235-2.328 $\mu_{B}$ with four sulfur substitutions, which are far below the expected saturation value for $S=3 / 2$ ion at $7 \mathrm{~T}$ and $2.0 \mathrm{~K}\left(M_{\mathrm{sat}}=3.88 \mu_{B}\right)$ and again is evidence of a large zero-field splitting. ${ }^{43}$

\section{Concluding Remarks}

The new proposed single-ion $\mathrm{Co}$ (II) complexes built from 1,2-dicarba-closo-dodecaborane and 1,2-dicarba-closo-hexaborane present similar distorted tetrahedral structure, but their magnetic properties turn out to be strikingly different. In all cases it has been verified through CASSCF calculations that their ground states are high-spin $(S=3 / 2)$ quartets. Including spin-orbit coupling through NEVPT2 calculations predict axial anisotropies of some of these complexes that are as large as $|D| \sim 140 \mathrm{~cm}^{-1}$, twice the $D$ measure in a closely related parent complex. ${ }^{22}$ For mononuclear Co(II) complexes derived from 1,2-dicarba-closododecaborane, substituting sulfur by oxygen favors an increase in the $D$ parameters, only when the carbon atoms are close to the Co ion, while for complexes derived from 1,2dicarba-closo-hexaborane, substituting sulfur by oxygen increases the parameter $D$ only when the octahedral ligands contain carbon atoms in the periphery. We found that for isomers with the same local $\mathrm{Co}(\mathrm{II})$ environment, there is an empirical relation that allows one to sort the complexes by their magnetic anisotropy using the structural parameters $\sigma(R)$ and $d$ from the MBE analysis. This relation could be used to identify mononuclear Co(II) complexes with large magnetic anisotropy. Our results provide evidence that o-carboraneincorporated $\mathrm{Co}(\mathrm{II})$ complexes are worthwhile candidates for experimental exploration as single-ion molecular magnets. 


\section{Supporting Information Available}

Summary of the main geometrical features, optimized structures, g-tensor components, excitation energies, temperature-dependent magnetic susceptibility, and field-dependent molar magnetization for all complexes in this work.

\section{Acknowledgements}

This work has been financially supported by the grants PCB No. 2013-1401PCB, PIP No. 11220130100377CO, and 11220130100311CO (Consejo Nacional de Investigaciones Científicas y Técnicas, Argentina), UBACYT 20020150100157BA (Universidad de Buenos Aires, Argentina), PICT No. 201-0381 (Agencia Nacional de Promoción Científica y Tecnológica, Argentina). JEP acknowledges support from the Office of Basic Energy Sciences, US Department of Energy, DE-SC0005027. 
Table 1: Parameters of the effective spin Hamiltonian $\hat{H}^{e f f}\left(D\right.$ in $\left.\mathrm{cm}^{-1}\right)$ and main g-tensor values of the Ground-State Kramers Doublet (gGKD) from CASSCF/NEVPT2 calculations on complexes with 1,2-dicarba-closo-dodecaborane ligands at the DFT (BP86) optimized geometries

\begin{tabular}{|c|c|c|c|c|c|c|c|c|}
\hline complex & \multicolumn{6}{|c|}{$\hat{H}^{\text {eff }}$ parameters } & \multicolumn{3}{c|}{ gGKD } \\
\hline & $g_{1}$ & $g_{2}$ & $g_{3}$ & $D$ & $E / D$ & $g_{1}$ & $g_{2}$ & $g_{3}$ \\
\hline $\mathrm{A}_{0}$ & 2.07 & 2.07 & 2.91 & -72.85 & 0.0016 & 0.0091 & 0.0105 & 8.6519 \\
$\mathrm{~B}_{0}$ & 1.47 & 1.49 & 3.52 & -147.22 & 0.0009 & 0.0036 & 0.0037 & 9.8242 \\
$\mathrm{C}_{0}$ & 2.19 & 2.20 & 2.71 & -43.53 & 0.0094 & 0.0594 & 0.0642 & 8.0869 \\
\hline $\mathrm{A}_{2}$ & 1.67 & 1.69 & 3.49 & -142.62 & 0.0035 & 0.0162 & 0.0163 & 9.8040 \\
$\mathrm{~B}_{2}$ & 2.07 & 2.08 & 3.18 & -99.50 & 0.0024 & 0.0141 & 0.0157 & 9.3221 \\
$\mathrm{~B}_{2}^{\prime}$ & 2.08 & 2.21 & 2.87 & -63.46 & 0.0866 & 0.5115 & 0.5939 & 8.4717 \\
$\mathrm{C}_{2}$ & 2.15 & 2.25 & 2.67 & -39.97 & 0.1105 & 0.6603 & 0.7755 & 7.8991 \\
\hline $\mathrm{A}_{4}$ & 2.10 & 2.12 & 3.10 & -88.19 & 0.0080 & 0.0499 & 0.0515 & 9.1348 \\
$\mathrm{~B}_{4}$ & 2.13 & 2.26 & 2.80 & -52.34 & 0.0982 & 0.5882 & 0.6900 & 8.2757 \\
$\mathrm{C}_{4}$ & 2.21 & 2.21 & 2.67 & -38.73 & 0.0011 & 0.0037 & 0.0106 & 7.9857 \\
\hline
\end{tabular}


Table 2: $\delta$ and $\Delta$ (in $\mathrm{cm}^{-1}$ ) are the computed first excitation energies for complexes with 1,2dicarba-closo-dodecaborane ligands arising from the CASSCF/NEVPT2 method before and after including the spin-orbit effects, respectively. The $\Delta$ value corresponds to the energy difference between the ground and the first excited Kramers doublets

\begin{tabular}{|c|c|c|}
\hline complex & \multicolumn{2}{|c|}{ CASSCF/NEVPT2 } \\
\hline & $\delta$ & $\Delta$ \\
\hline $\mathrm{A}_{0}$ & 1049.0 & $145.7 / 1243.0$ \\
$\mathrm{~B}_{0}$ & 68.5 & $294.4 / 635.5$ \\
$\mathrm{C}_{0}$ & 1548.0 & $87.1 / 1661.2$ \\
\hline $\mathrm{A}_{2}$ & 167.6 & $285.3 / 659.2$ \\
$\mathrm{~B}_{2}$ & 606.3 & $199.0 / 887.3$ \\
$\mathrm{~B}_{2}^{\prime}$ & 1115.0 & $128.3 / 1287.0$ \\
$\mathrm{C}_{2}$ & 1657.8 & $81.4 / 1758.0$ \\
\hline $\mathrm{A}_{4}$ & 747.5 & $176.4 / 992.8$ \\
$\mathrm{~B}_{4}$ & 1305.4 & $106.2 / 1445.5$ \\
$\mathrm{C}_{4}$ & 1699.2 & $77.5 / 1798.5$ \\
\hline
\end{tabular}


Table 3: Ellipsoidal analysis of coordination polyhedra for the complexes with 1,2-dicarbacloso-dodecaborane ligands from DFT (BP86) geometry optimization

\begin{tabular}{|c|c|c|c|c|c|}
\hline $\begin{array}{c}\text { No. S atoms } \\
\text { substituted }\end{array}$ & & $\mathrm{A}$ & \multicolumn{2}{|c|}{$\mathrm{B}$} & $\mathrm{C}$ \\
\hline \multirow{7}{*}{0} & $R_{1}$ & 2.6513 & \multicolumn{2}{|c|}{2.6475} & 2.5502 \\
\hline & $R_{2}$ & 2.0612 & \multicolumn{2}{|c|}{2.1680} & 2.2682 \\
\hline & $R_{3}$ & 2.0323 & \multicolumn{2}{|c|}{2.0600} & 2.2229 \\
\hline & $\langle R\rangle$ & 2.2483 & \multicolumn{2}{|c|}{2.2918} & 2.3471 \\
\hline & $\sigma(R)$ & 0.2852 & \multicolumn{2}{|c|}{0.2553} & 0.1448 \\
\hline & $\mathrm{S}$ & 0.2085 & \multicolumn{2}{|c|}{0.1313} & 0.0906 \\
\hline & $d$ & 0.0185 & \multicolumn{2}{|c|}{0.0303} & 0.0059 \\
\hline \multirow{7}{*}{2} & $R_{1}$ & 2.5705 & 2.5617 & 2.4711 & 2.4350 \\
\hline & $R_{2}$ & 2.0573 & 2.1779 & 2.1273 & 2.2452 \\
\hline & $R_{3}$ & 1.7002 & 1.7159 & 1.8039 & 1.8318 \\
\hline & $\langle R\rangle$ & 2.1093 & 2.1518 & 2.1341 & 2.1707 \\
\hline & $\sigma(R)$ & 0.3572 & 0.3458 & 0.2724 & 0.2518 \\
\hline & $\mathrm{S}$ & 0.0261 & -0.0623 & -0.0129 & -0.1062 \\
\hline & $d$ & 0.0304 & 0.0161 & 0.1145 & 0.0667 \\
\hline \multirow{7}{*}{4} & $R_{1}$ & 2.4343 & \multicolumn{2}{|c|}{2.3837} & 2.3324 \\
\hline & $R_{2}$ & 1.7733 & \multicolumn{2}{|c|}{1.8327} & 1.8432 \\
\hline & $R_{3}$ & 1.7112 & \multicolumn{2}{|c|}{1.7468} & 1.8397 \\
\hline & $\langle R\rangle$ & 1.9729 & \multicolumn{2}{|c|}{1.9877} & 2.0051 \\
\hline & $\sigma(R)$ & 0.3272 & \multicolumn{2}{|c|}{0.2822} & 0.2314 \\
\hline & $\mathrm{S}$ & 0.2366 & \multicolumn{2}{|c|}{0.1843} & 0.2079 \\
\hline & $d$ & 0.0150 & \multicolumn{2}{|c|}{0.0758} & 0.0051 \\
\hline
\end{tabular}


Table 4: Magnetization, $M$, at $2.0 \mathrm{~K}$ and $7 \mathrm{~T}$ and values of $\chi T$ at $3.0 \mathrm{~K}$ and $300.0 \mathrm{~K}$ and 1000 Oe of 1,2-dicarba-closo-dodecaboranes

\begin{tabular}{|c|c|c|c|}
\hline complex & \multicolumn{2}{|c|}{$\chi T$} & $M$ \\
\hline & $3.0 \mathrm{~K}$ & $300.0 \mathrm{~K}$ & $7 \mathrm{~T}$ \\
\hline $\mathrm{A}_{0}$ & 2.361 & 2.972 & 2.246 \\
$\mathrm{~B}_{0}$ & 3.024 & 3.571 & 2.494 \\
$\mathrm{C}_{0}$ & 2.087 & 2.875 & 2.181 \\
\hline $\mathrm{A}_{2}$ & 3.012 & 3.570 & 2.492 \\
$\mathrm{~B}_{2}$ & 2.731 & 3.386 & 2.394 \\
$\mathrm{~B}_{2}^{\prime}$ & 2.290 & 3.012 & 2.254 \\
$\mathrm{C}_{2}$ & 2.030 & 2.850 & 2.204 \\
\hline $\mathrm{A}_{4}$ & 2.626 & 3.304 & 2.356 \\
$\mathrm{~B}_{4}$ & 2.202 & 3.001 & 2.245 \\
$\mathrm{C}_{4}$ & 2.042 & 2.861 & 2.181 \\
\hline
\end{tabular}


Table 5: Parameters of the effective spin Hamiltonian $\hat{H}^{e f f}\left(D\right.$ in $\left.\mathrm{cm}^{-1}\right)$ and main g-tensor values of the Ground-State Kramers Doublet (gGKD) from CASSCF/NEVPT2 calculations on complexes with 1,2-dicarba-closo-hexaborane ligands at the DFT (BP86) optimized geometries

\begin{tabular}{|c|c|c|c|c|c|c|c|c|}
\hline complex & \multicolumn{7}{|c|}{$\hat{H}^{\text {eff }}$ parameters } & \multicolumn{3}{c|}{ gGKD } \\
\hline & $g_{1}$ & $g_{2}$ & $g_{3}$ & $D$ & $E / D$ & $g_{1}$ & $g_{2}$ & $g_{3}$ \\
\hline $\mathrm{A}_{0}$ & 1.64 & 1.65 & 3.50 & -144.57 & 0.0006 & 0.0027 & 0.0028 & 9.8119 \\
$\mathrm{~B}_{0}$ & 2.18 & 2.25 & 2.67 & -37.47 & 0.0788 & 0.4881 & 0.5505 & 7.9234 \\
$\mathrm{C}_{0}$ & 2.29 & 2.30 & 2.46 & -13.20 & 0.0209 & 0.1371 & 0.1508 & 7.3775 \\
\hline $\mathrm{A}_{2}$ & 2.08 & 2.17 & 3.01 & -78.20 & 0.0380 & 0.2329 & 0.2526 & 8.8909 \\
$\mathrm{~B}_{2}$ & 2.23 & 2.26 & 2.64 & -33.31 & 0.0409 & 0.2622 & 0.2868 & 7.8969 \\
$\mathrm{~B}_{2}^{\prime}$ & 2.15 & 2.29 & 2.60 & -31.48 & 0.2081 & 1.1338 & 1.4845 & 7.4669 \\
$\mathrm{C}_{2}$ & 2.23 & 2.35 & 2.47 & 15.94 & 0.2710 & 1.7994 & 2.7590 & 6.5143 \\
\hline $\mathrm{A}_{4}$ & 2.15 & 2.18 & 2.90 & -63.95 & 0.0183 & 0.1180 & 0.1184 & 8.6195 \\
$\mathrm{~B}_{4}$ & 2.20 & 2.29 & 2.60 & -29.83 & 0.1386 & 0.8235 & 0.9920 & 7.6383 \\
$\mathrm{C}_{4}$ & 2.28 & 2.28 & 2.48 & -16.17 & 0.0132 & 0.0832 & 0.0973 & 7.4357 \\
\hline
\end{tabular}


Table 6: Ellipsoidal analysis of coordination polyhedra for the complexes with 1,2-dicarbacloso-hexaborane ligands from DFT (BP86) geometry optimization.

\begin{tabular}{|c|c|c|c|c|c|}
\hline $\begin{array}{c}\text { No. S atoms } \\
\text { substituted }\end{array}$ & & $\mathrm{A}$ & \multicolumn{2}{|c|}{$\mathrm{B}$} & $\mathrm{C}$ \\
\hline \multirow{7}{*}{0} & $R_{1}$ & 2.7302 & \multicolumn{2}{|c|}{2.5640} & 2.4457 \\
\hline & $R_{2}$ & 2.1131 & \multicolumn{2}{|c|}{2.4057} & 2.4124 \\
\hline & $R_{3}$ & 2.0635 & \multicolumn{2}{|c|}{2.1467} & 2.3940 \\
\hline & $\langle R\rangle$ & 2.3023 & \multicolumn{2}{|c|}{2.3721} & 2.4173 \\
\hline & $\sigma(R)$ & 0.3033 & \multicolumn{2}{|c|}{0.1720} & 0.0214 \\
\hline & $\mathrm{S}$ & 0.2025 & \multicolumn{2}{|c|}{-0.0459} & 0.0060 \\
\hline & $d$ & 0.0026 & \multicolumn{2}{|c|}{0.0823} & 0.0027 \\
\hline \multirow{7}{*}{2} & $R_{1}$ & 2.5713 & 2.4371 & 2.4477 & 2.4080 \\
\hline & $R_{2}$ & 2.1681 & 2.4081 & 2.1575 & 2.3247 \\
\hline & $R_{3}$ & 1.7578 & 1.7773 & 1.9981 & 2.0047 \\
\hline & $\langle R\rangle$ & 2.1658 & 2.2075 & 2.2011 & 2.2458 \\
\hline & $\sigma(R)$ & 0.3321 & 0.3044 & 0.1861 & 0.1739 \\
\hline & S & -0.0324 & -0.2501 & 0.0446 & -0.1030 \\
\hline & $d$ & 0.0623 & 0.0259 & 0.1487 & 0.0673 \\
\hline \multirow{7}{*}{4} & $R_{1}$ & 2.4658 & \multicolumn{2}{|c|}{2.3303} & 2.2080 \\
\hline & $R_{2}$ & 1.8296 & \multicolumn{2}{|c|}{2.0170} & 2.0206 \\
\hline & $R_{3}$ & 1.7188 & \multicolumn{2}{|c|}{1.7762} & 2.0075 \\
\hline & $\langle R\rangle$ & 2.0047 & \multicolumn{2}{|c|}{2.0412} & 2.0787 \\
\hline & $\sigma(R)$ & 0.3291 & \multicolumn{2}{|c|}{0.2269} & 0.0916 \\
\hline & S & 0.1975 & \multicolumn{2}{|c|}{0.0150} & 0.0783 \\
\hline & $d$ & 0.0008 & \multicolumn{2}{|c|}{0.0951} & 0.0050 \\
\hline
\end{tabular}


Table 7: $\delta$ and $\Delta$ (in $\mathrm{cm}^{-1}$ ) are the computed first excitation energies for complexes with 1,2-dicarba-closo-hexaborane ligands arising from the CASSCF/NEVPT2 method before and after including the spin-orbit effects, respectively. The $\Delta$ value corresponds to the energy difference between the ground and the first excited Kramers doublets

\begin{tabular}{|c|c|c|}
\hline complex & \multicolumn{2}{|c|}{ CASSCF/NEVPT2 } \\
\hline & $\delta$ & $\Delta$ \\
\hline $\mathrm{A}_{0}$ & 146.2 & $289.1 / 655.0$ \\
$\mathrm{~B}_{0}$ & 1648.5 & $75.6 / 1733.3$ \\
$\mathrm{C}_{0}$ & 2550.9 & $26.4 / 2543.6$ \\
\hline $\mathrm{A}_{2}$ & 881.2 & $156.7 / 1096.4$ \\
$\mathrm{~B}_{2}$ & 1759.4 & $66.8 / 1822.8$ \\
$\mathrm{~B}_{2}^{\prime}$ & 1887.5 & $66.9 / 1966.7$ \\
$\mathrm{C}_{2}$ & 2540.6 & $35.2 / 2539.1$ \\
\hline $\mathrm{A}_{4}$ & 1110.1 & $128.0 / 1282.5$ \\
$\mathrm{~B}_{4}$ & 1923.6 & $61.4 / 1990.4$ \\
$\mathrm{C}_{4}$ & 2498.2 & $32.3 / 2509.7$ \\
\hline
\end{tabular}


Table 8: Magnetization, $M$, at $2.0 \mathrm{~K}$ and $7 \mathrm{~T}$ and values of $\chi T$ at $3.0 \mathrm{~K}$ and $300.0 \mathrm{~K}$ and at 1000 Oe of 1,2-dicarba-closo-hexaboranes

\begin{tabular}{|c|c|c|c|}
\hline complex & \multicolumn{2}{|c|}{$\chi T$} & $M$ \\
\hline & $3.0 \mathrm{~K}$ & $300.0 \mathrm{~K}$ & $7 \mathrm{~T}$ \\
\hline $\mathrm{A}_{0}$ & 3.016 & 3.560 & 2.495 \\
$\mathrm{~B}_{0}$ & 2.030 & 2.865 & 2.203 \\
$\mathrm{C}_{0}$ & 1.861 & 2.771 & 2.423 \\
\hline $\mathrm{A}_{2}$ & 2.495 & 3.182 & 2.315 \\
$\mathrm{~B}_{2}$ & 2.013 & 2.881 & 2.206 \\
$\mathrm{~B}_{2}^{\prime}$ & 1.913 & 2.796 & 2.242 \\
$\mathrm{C}_{2}$ & 1.792 & 2.770 & 2.435 \\
\hline $\mathrm{A}_{4}$ & 2.350 & 3.085 & 2.266 \\
$\mathrm{~B}_{4}$ & 1.941 & 2.827 & 2.235 \\
$\mathrm{C}_{4}$ & 1.856 & 2.760 & 2.328 \\
\hline
\end{tabular}




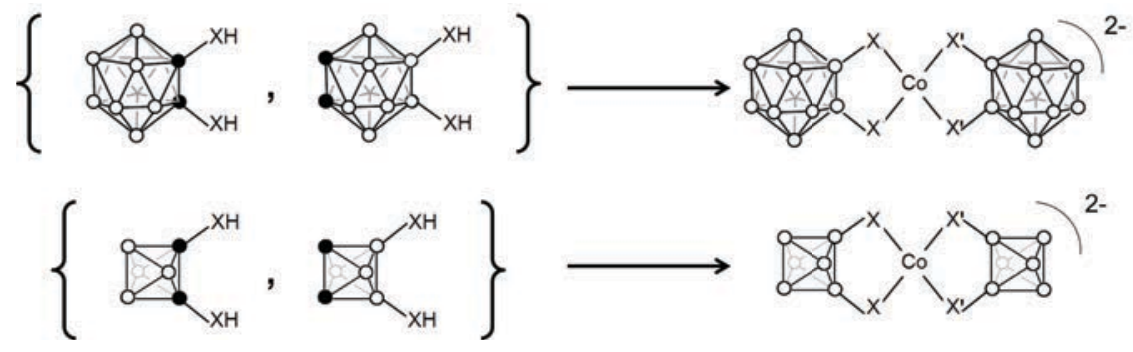

Figure 1: Scheme of thiolated and hydroxylated positional isomers 1,2-dicarba-closododecaborane and 1,2-dicarba-closo-hexaborane: 1,2-( $\mathrm{HX})_{2}-1,2-\mathrm{C}_{2} \mathrm{~B}_{10} \mathrm{H}_{10}$ and 9,12-( $\left.\mathrm{HX}\right)_{2}$ $1,2-\mathrm{C}_{2} \mathrm{~B}_{10} \mathrm{H}_{10}, 1,2-(\mathrm{HX})_{2}-1,2-\mathrm{C}_{2} \mathrm{~B}_{4} \mathrm{H}_{4}$ and 4,6-(HX) $)_{2}-1,2-\mathrm{C}_{2} \mathrm{~B}_{4} \mathrm{H}_{4}(\mathrm{X}: \mathrm{S}, \mathrm{O})$ and formation of complexes. 


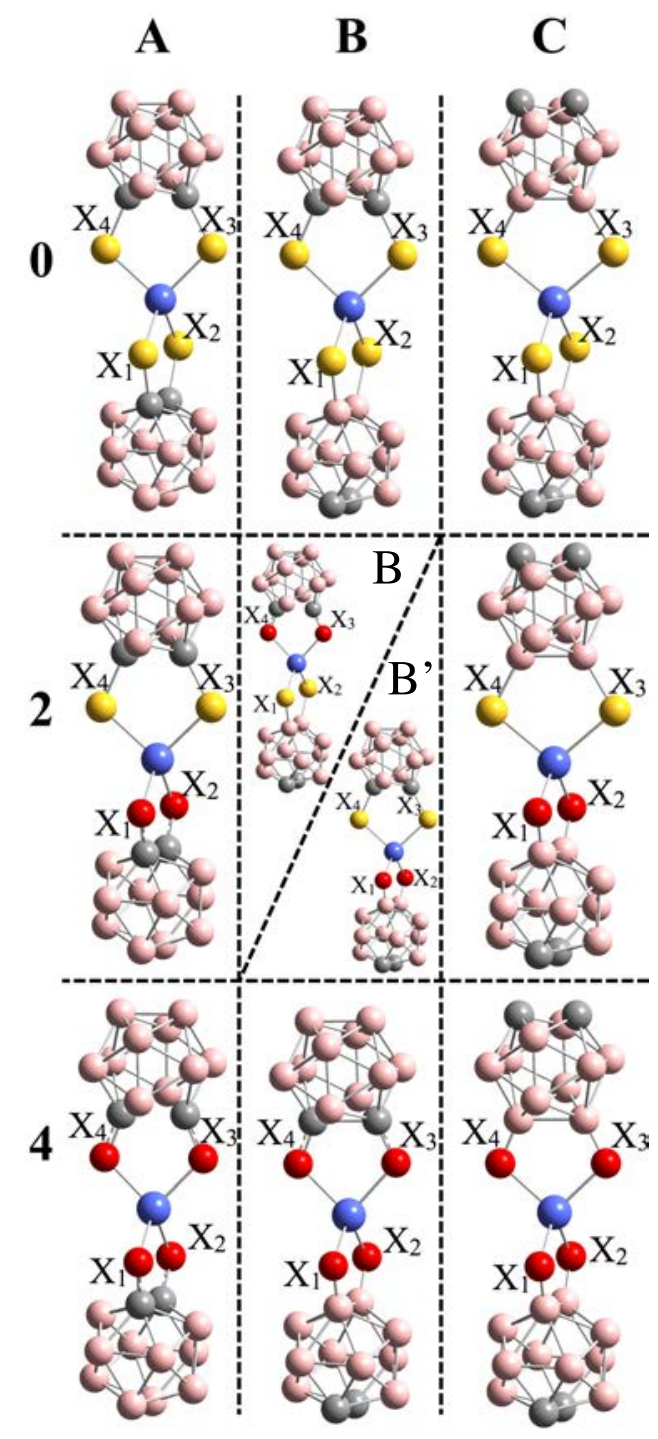

Figure 2: Molecular structure of $\mathrm{Co}$ (II) 1,2-dicarba-closo-dodecaborane complexes including bridging atom labeling $\mathrm{X}_{1}, \mathrm{X}_{2}, \mathrm{X}_{3}$, and $\mathrm{X}_{4}$. Hydrogen atoms were omitted for clarity. Oxygen atoms are in red color, sulfur in yellow, boron in pink, carbon in gray, and cobalt in blue. 


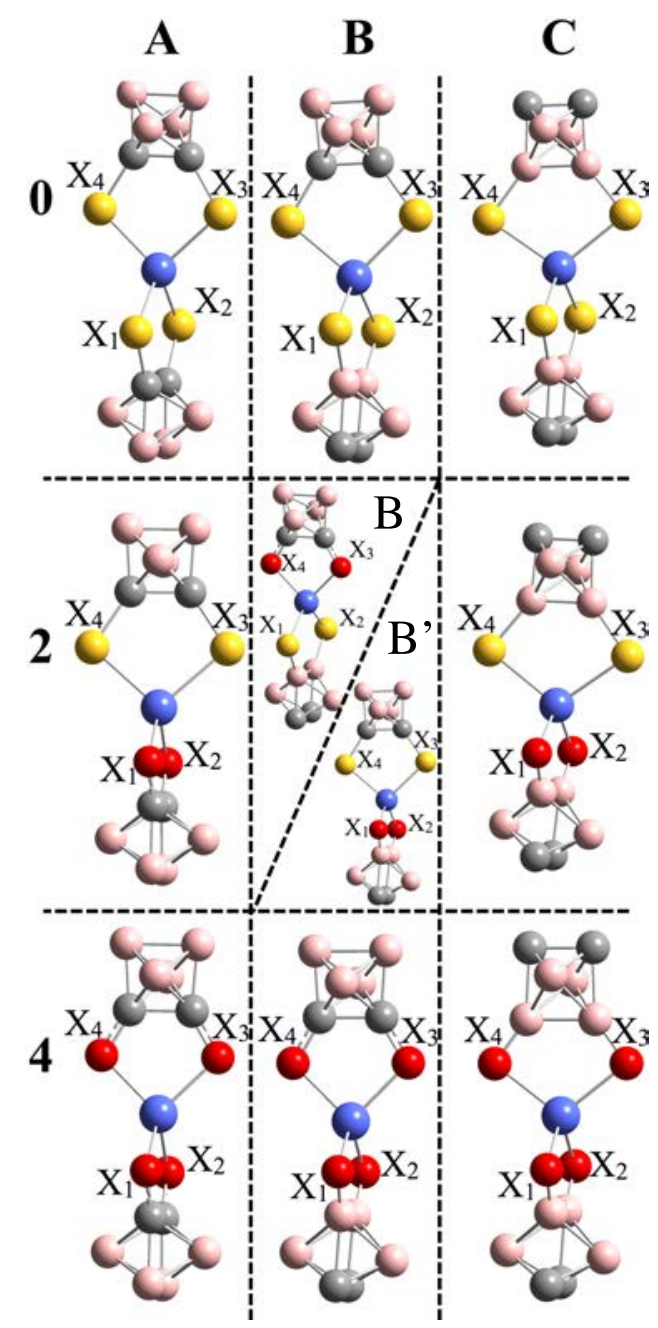

Figure 3: Molecular structure of $\mathrm{Co}$ (II) 1,2-dicarba-closo-hexaborane complexes including bridging atom labeling $\mathrm{X}_{1}, \mathrm{X}_{2}, \mathrm{X}_{3}$, and $\mathrm{X}_{4}$. Hydrogen atoms were omitted for clarity. Oxygen atoms are in red color, sulfur in yellow, boron in pink, carbon in gray, and cobalt in blue.

\section{References}

(1) Kahn, O. Molecular Magnetism, 1st ed.; VCH Publishers, Inc., New York, 1993.

(2) Wernsdorfer, W.; Sessoli, R. Quantum Phase Interference and Parity Effects in Magnetic Molecular Clusters. Science 1999, 284, 133-135.

(3) Ishikawa, N.; Sugita, M.; Ishikawa, T.; Koshihara, S.; Kaizu, Y. Lanthanide Double- 
Decker Complexes Functioning as Magnets at the Single-Molecular Level. J. Am. Chem. Soc. 2003, 125, 8694-8695.

(4) Miller, J. S. Magnetically Ordered Molecule-based Materials. Chem. Soc. Rev. 2011, 40, 3266-3296.

(5) Sugawara, T.; Matsushita, M. M. Spintronics in Organic $\pi$-electronic Systems. J. Mater. Chem. 2009, 19, 1738-1753.

(6) Ratera, I.; Veciana, J. Playing with Organic Radicals as Building Blocks for Functional Molecular Materials. Chem. Soc. Rev. 2012, 41, 303-349.

(7) Ganzhorn, M.; Wernsdorfer, W. In Molecular Magnets; Bartolome, J., Luis, F., Fernandez, J., Eds.; Springer, Berlin, 2014.

(8) Atanasov, M.; Aravena, D.; Suturina, E.; Bill, E.; Maganas, D.; Neese, F. First Principles Approach to the Electronic Structure, Magnetic Anisotropy and Spin Relaxation in Mononuclear 3d-transition Metal Single Molecule Magnets. Coord. Chem. Rev. 2015, 289-290, 177-214.

(9) Sessoli, R.; Gatteschi, D.; Caneschi, A.; Novak, M. A. Magnetic Bistability in a Metalion Cluster. Nature 1993, 365, 141-143.

(10) Gatteschi, D.; Sessoli, R.; Villain, J. Molecular Nanomagnets.; Oxford University Press, New York, 2006.

(11) Christou, G.; Gatteschi, D.; Hendrickson, D. N.; Sessoli, R. Single-Molecule Magnets. MRS Bulletin 2000, 25, 66-71.

(12) Neese, F.; Pantazis, D. A. What is not required to make a single molecule magnet. Faraday Discuss. 2011, 148, 229-238. 
(13) Maganas, D.; Sottini, S.; Kyritsis, P.; Groenen, E. J. J.; Neese, F. Theoretical Analysis of the Spin Hamiltonian Parameters in $\mathrm{Co}(\mathrm{II}) \mathrm{S}_{4}$ Complexes, Using Density Functional Theory and Correlated ab initio Methods. Inorg. Chem. 2011, 50, 8741-8754.

(14) Fataftah, M. S.; Zadrozny, J. M.; Rogers, D. M.; Freedman, D. E. A Mononuclear Transition Metal Single-Molecule Magnet in a Nuclear Spin-Free Ligand Environment. Inorg. Chem. 2014, 53, 10716-10721.

(15) King, B. T.; Noll, B. C.; McKinley, A. J.; Michl, J. Dodecamethylcarba-closododecaboranyl $\left(\mathrm{CB}_{11} \mathrm{Me}_{12}{ }^{\circ}\right)$, a Stable Free Radical. J. Am. Chem. Soc. 1996, 118, 10902-10903.

(16) Hnyk, D.; McKee, M. Boron: The Fifth Element, in Challenges and Advances in Computational Chemistry and Physics 20; Springer, Dordrecht, 2015.

(17) Grimes, R. N. Carboranes, 3rd ed.; Academic Press, New York, 2016.

(18) Oliva, J. M.; Alcoba, D. R.; Lain, L.; Torre, A. Electronic Structure Studies of Diradicals Derived from Closo-Carboranes. Theor. Chem. Acc. 2013, 132, 1329.

(19) Oliva, J. M.; Alcoba, D. R.; Oña, O. B.; Torre, A.; Lain, L.; Michl, J. Toward (Car)Borane-based Molecular Magnets. Theor. Chem. Acc. 2015, 134, 9.

(20) Alcoba, D. R.; Oña, O. B.; Massaccesi, G. E.; Torre, A.; Lain, L.; Notario, R.; Oliva, J. M. Molecular Magnetism in Closo-azadodecaborane Supericosahedrons. Mol. Phys. 2016, 114, 400-406.

(21) Oña, O. B.; Alcoba, D. R.; Torre, A.; Lain, L.; Massaccesi, G. E.; Oliva-Enrich, J. M. Determination of Exchange Coupling Constants in Linear Polyradicals by means of Local Spins. Theor. Chem. Acc. 2017, 136, 35. 
(22) Tu, D.; Shao, D.; Yan, H.; Lu, C. A Carborane-Incorporated Mononuclear Co(ii) Complex Showing Zero-Field Slow Magnetic Relaxation. Chem. Commun. 2016, 52, 1432614329.

(23) Alcoba, D. R.; Oña, O. B.; Massaccesi, G. E.; Torre, A.; Lain, L.; Melo, J. I.; Peralta, J. E.; Oliva-Enrich, J. M. Magnetic Properties of Mononuclear Co(II) Complexes with Carborane Ligands. Inorg. Chem. 2018, 57, 7763-7769.

(24) Plešek, J.; Heřmánek, S. Experimental evaluation of charge distribution on particular skeletal atoms in icosahedral carboranes by means of HS-derivatives. Collect. Czech. Chem. Commun. 1979, 44, 24-33.

(25) Plešek, J.; Heřánek, S. Synthesis and properties of some icosahedral carborane B,B'dithiols. Collect. Czech. Chem. Commun. 1980, 45, 1775-1779.

(26) Zakharkin, L.; Pisareva, I. Synthesis of 9,12-o- and 9,10-m-carboranyl-dithiols and diselenols from $\mathrm{S}_{2} \mathrm{Cl}_{2}$ or $\mathrm{Se}_{2} \mathrm{Cl}_{2}$ and o- and m-carboranes. J. Organomet. Chem. 1984, 267, 73-79.

(27) Ohta, K.; Goto, T.; Yamazaki, H.; Pichierri, F.; Endo, Y. Facile and Efficient Synthesis of C-Hydroxycarboranes and C,C-Dihydroxycarboranes. Inorg. Chem. 2007, 46, 39663970.

(28) Oliva, J. M.; Serrano-Andrés, L. A computational study of the lowest singlet and triplet states of neutral and dianionic 1,2-substituted icosahedral and octahedral o-carboranes. J. Comput. Chem. 2006, 27, 524-535.

(29) Novikov, V. V.; Pavlov, A. A.; Nelyubina, Y. V.; Boulon, M.-E.; Varzatskii, O. A.; Voloshin, Y. Z.; Winpenny, R. E. P. A Trigonal Prismatic Mononuclear Cobalt(II) Complex Showing Single-Molecule Magnet Behavior. J. Am. Chem. Soc. 2015, 137, 9792-9795. 
(30) Postnikov, A. V.; Kortus, J.; Pederson, M. R. Density functional studies of molecular magnets. Phys. Status Solidi B 2006, 243, 2533-2572.

(31) Cumby, J.; Attfield, J. P. Ellipsoidal Analysis of Coordination Polyhedra. Nature Communications 2017, 8, 14235.

(32) Perdew, J. P. Density-functional approximation for the correlation energy of the inhomogeneous electron gas. Phys. Rev. B 1986, 33, 8822-8824.

(33) Becke, A. D. Density-Functional Exchange-Energy Approximation with Correct Asymptotic Behavior. Phys. Rev. A 1988, 38, 3098-3100.

(34) Schäfer, A.; Horn, H.; Ahlrichs, R. Fully optimized contracted Gaussian basis sets for atoms Li to Kr. J. Chem. Phys. 1992, 97, 2571-2577.

(35) Vahtras, O.; Almlöf, J.; Feyereisen, M. Integral Approximations for LCAO-SCF Calculations. Chem. Phys. Lett. 1993, 213, $514-518$.

(36) Weigend, F.; Ahlrichs, R. Balanced basis sets of split valence, triple zeta valence and quadruple zeta valence quality for H to Rn: Design and assessment of accuracy. Phys. Chem. Chem. Phys. 2005, 7, 3297-3305.

(37) Angeli, C.; Cimiraglia, R.; Malrieu, J.-P. N-electron valence state perturbation theory: a fast implementation of the strongly contracted variant. Chem. Phys. Lett. 2001, 350, $297-305$.

(38) Angeli, C.; Cimiraglia, R.; Evangelisti, S.; Leininger, T.; Malrieu, J.-P. Introduction of n-electron valence states for multireference perturbation theory. J. Chem. Phys. 2001, $114,10252-10264$.

(39) Angeli, C.; Cimiraglia, R.; Malrieu, J.-P. n-electron valence state perturbation theory: A spinless formulation and an efficient implementation of the strongly contracted and of the partially contracted variants. J. Chem. Phys. 2002, 117, 9138-9153. 
(40) Pierloot, K.; Phung, Q. M.; Domingo, A. Spin State Energetics in First-Row Transition Metal Complexes: Contribution of (3s3p) Correlation and Its Description by SecondOrder Perturbation Theory. J. Chem. Theory Comput. 2017, 13, 537-553.

(41) Neese, F. The ORCA program system. Wiley Interdisciplinary Reviews: Comput. Mol. Sci. 2012, 2, 73-78.

(42) Todd, M. J.; Yildirim, E. A. On Khachiyan's Algorithm for the Computation of Minimum-volume Enclosing Ellipsoids. Discrete Appl. Math. 2007, 155, 1731-1744.

(43) Rechkemmer, Y.; Breitgoff, F. D.; van der Meer, M.; Atanasov, M.; Hakl, M.; Orlita, M.; Neugebauer, P.; Neese, F.; Sarkar, B.; van Slageren, J. A Four-Coordinate Cobalt(II) Single-ion Magnet with Coercivity and a Very High Energy Barrier. Nat. Comm. 2016, 7, 10467. 


\section{Graphical TOC Entry}

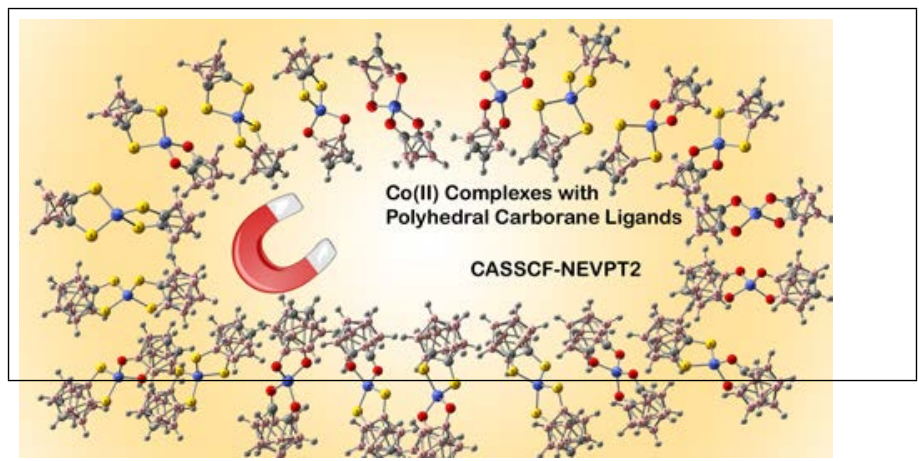

Magnetic Properties of $20 \mathrm{Co}$ (II) Complexes with Polyhedral Carborane Ligands. 